\title{
Long-term neurological manifestations of COVID-19: prevalence and predictive factors
}

\author{
Andrea Pilotto ${ }^{1,2}$ (D) Viviana Cristillo ${ }^{1}$ - Stefano Cotti Piccinelli ${ }^{1}$ - Nicola Zoppi ${ }^{1}$ - Giulio Bonzi ${ }^{1}$ • Davide Sattin ${ }^{2}$. \\ Silvia Schiavolin ${ }^{2} \cdot$ Alberto Raggi $^{2}$ - Antonio Canale ${ }^{3} \cdot$ Stefano Gipponi $^{1} \cdot$ Ilenia Libri ${ }^{1}$ - Martina Frigerio ${ }^{1}$. \\ Michela Bezzi ${ }^{4} \cdot$ Matilde Leonardi $^{2} \cdot$ Alessandro Padovani $^{1}$
}

Received: 2 May 2021 / Accepted: 26 August 2021 / Published online: 15 September 2021

(C) The Author(s) 2021, corrected publication 2022

\begin{abstract}
Background Clinical investigations have argued for long-term neurological manifestations in both hospitalised and non-hospitalised COVID-19 patients. It is unclear whether long-term neurological symptoms and features depend on COVID-19 severity.

Methods From a sample of 208 consecutive non-neurological patients hospitalised for COVID-19 disease, 165 survivors were re-assessed at 6 months according to a structured standardised clinical protocol. Prevalence and predictors of long-term neurological manifestations were evaluated using multivariate logistic regression analyses.

Results At 6-month follow-up after hospitalisation due to COVID-19 disease, patients displayed a wide array of symptoms; fatigue (34\%), memory/attention (31\%) and sleep disorders (30\%) were the most frequent. At neurological examination, $40 \%$ of patients exhibited neurological abnormalities, such as hyposmia (18.0\%), cognitive deficits (17.5\%), postural tremor $(13.8 \%)$ and subtle motor/sensory deficits (7.6\%). Older age, premorbid comorbidities and severity of COVID-19 were independent predictors of neurological manifestations in logistic regression analyses.

Conclusions Premorbid vulnerability and severity of SARS-CoV-2 infection impact on prevalence and severity of long-term neurological manifestations.
\end{abstract}

Keywords COVID-19 $\cdot$ Fatigue $\cdot$ Hyposmia $\cdot$ Depression $\cdot$ Cognitive impairment

\section{Introduction}

After the first cases of the novel coronavirus disease 2019 (COVID-19) were reported in Wuhan, China, in December 2019, the spread rapidly in Europe became a pandemic, involving millions of cases worldwide [1]. With the increasing number of confirmed cases and the accumulating clinical data, it is now

Andrea Pilotto

pilottoandreae@gmail.com

Alessandro Padovani

alessandro.padovani@unibs.it

1 Neurology Unit, Department of Clinical and Experimental Sciences, University of Brescia, P.le Spedali Civili 1, 25123 Brescia, Italy

2 Neurology, Public Health Disability Unit - Fondazione IRCCS Istituto Neurologico Carlo Besta, Milan, Italy

3 Department of Statistics, University of Padova, Padua, Italy

4 Respiratory Unit, ASST Spedali Civili di Brescia, Brescia, Italy well established that, in addition to the predominant respiratory symptoms, a significant proportion of COVID-19 patients experience neurological symptoms and syndromes [2-5]. Clinical findings on previously hospitalised and non-hospitalised patients with COVID-19 reported the persistence of multiple symptoms, particularly fatigue, dyspnoea, sleep disturbances and memory complaints syndromes [6-8]. Accordingly, some authors have suggested the so-called, but not yet defined, post-COVID-19 syndrome based on persistent symptoms reported after resolution of SARS-CoV-2 infection [8]. To date, however, the prevalence and severity of neurological long-term manifestations and the correlation with severity of COVID-19 infection are still under debate, as no long-term data using extensive neurological assessment are still available yet in the growing literature.

In this study, subjects previously hospitalised for COVID19 disease entered a longitudinal study in order to evaluate general and neurological manifestations after 6 months of follow-up and their potential relationship with premorbid conditions and severity of respiratory infection. 


\section{Methods}

All patients who survived COVID-19 disease and were discharged between February and April 2020 from a COVID-19 Unit of the ASST Spedali Civili Brescia Hospital were asked to participate in a follow-up study. This includes a standardised evaluation of medical history, self-reported neurological symptoms and a complete neurological examination at 6 months. Premorbid conditions were recorded at admission using the Cumulative Illness Rating Scale [9] (CIRS). Patients with premorbid neurological conditions (including dementia) were excluded. Hospitalisation data included the severity of COVID-19 disease, classified according to the Brescia-COVID Respiratory Severity Scale (BCRSS), stratifying patients into mild, moderate and severe [10] and the quick Sequential Organ Failure Assessment (qSOFA) score [11].

At follow-up, data were collected using a structured questionnaire evaluating the presence of neurological symptoms related to central, peripheral, myopathic and cognitive manifestations via telemedicine (Supplementary Table 1). Each patient was additionally asked to undergo a complete neurological examination in the hospital in order to objectively assess cranial nerves, motor (global and focal), sensory, cerebellar, basal ganglia-related function, deep tendon reflexes, pyramidal signs. Global cognitive function was carried out by using the Montreal Cognitive Assessment (MoCA) by Italian validated norms [12]. The study was approved by the local ethics committee of ASST "Spedali Civili di Brescia" Hospital and the requirement for informed consent was waived by the Ethics Commission (NP 4166).

\section{Statistical analysis}

Differences between patients according to COVID-19 respiratory severity (BCRSS) and the association with neurological complaints were evaluated by Fisher's exact test or ANOVA with Bonferroni correction for dichotomic and continuous variables, respectively. To explore the risk factors associated with neurological symptoms and neurological features, univariable and multivariable logistic regression models were implemented.

Separate binary logistic regression analyses adjusted for age and sex evaluated the relationship between COVID-19 severity (moderate/severe vs mild-independent variable) with specific neurological symptoms (dependent variable). A multivariate linear regression model evaluated the relationship between total number of symptoms reported (dependent variable) and the following independent variable: age, sex, premorbid CIRS, days of hospitalisation and COVID-19 severity.

The relationship between the same independent variables and the presence of neurological abnormalities at the examination were first tested using univariate analyses and confirmed in multivariate logistic regression model. The strength of association between dependent and independent variables was evaluated using standard beta coefficient (beta) and $\operatorname{Exp}(B)$ for linear and logistic multivariable regression, respectively.

\section{Results}

From a sample of 208 consecutively hospitalised patients for COVID-19 disease, 33 deceased during hospitalisation. Survivors were younger $(p=0.001 ; 65.7 \pm 12.6$ vs $78.6 \pm 8.6)$ and exhibited less comorbidities $(p=0.004$, mean CIRS 1.36 \pm 0.51 vs $1.51 \pm 0.4)$ and lower COVID- 19 severity $(p=0.001$, mean BCRSS $0.90 \pm 0.85$ vs $2.89 \pm 0.32$ ) compared to deceased patients.

Out of 175 survivors, five patients died after discharge, three had a previous diagnosis of dementia and two refused to participate, resulting in a final sample of 165 patients (Supplementary Figure 1). Patients stratified according to COVID-19 severity (BCRSS) differ for the number of days of hospitalisation, $\mathrm{O} 2$ treatment and qSOFA but not for age or premorbid CIRS (Table 1).

At follow-up, no patients reported new hospitalisations or new onset of medical conditions. The most common symptoms reported were fatigue (34\%), memory complaints (31\%), sleep disorders $(30.8 \%)$ and myalgias $(29.6 \%)$, followed by depression/anxiety, visual disturbances, paraesthesia and hyposmia (Figure 1, Supplementary Table 2). Patients with moderate/severe COVID-19 reported higher number of symptoms at follow-up ( $p=0.004)$ after correction for age and premorbid CIRS. In univariable analyses, moderate/severe COVID-19 was associated with increased risk of memory complaints (OR 2.6, 95\% CI 1.18-5.8), loss of dependency in IADL (OR 2.6, 95\% CI 1.12-6.2), confusion (OR 2.9, 95\% CI 1.12-7.8), fatigue (OR 2.1, 95\% CI 0.95-4.6) and visual disturbances (OR 3.5, 95\% CI 1.5-8.4) at 6 months of follow-up compared to mild disease. Multivariable analyses identified premorbid comorbidities ( $p=0.006$, beta 0.26 ), age at admission ( $p=0.048$, beta 0.17 ) and severity of COVID-19 ( $p=0.04$, beta 0.22 ) as predictors of total number of symptoms reported.

One-hundred and five patients $(63.6 \%)$ were further evaluated using a standard neurological examination and cognitive screening. This group of patients were comparable to patients evaluated via telemedicine for age, sex distribution, premorbid CIRS, severity of COVID-19 and reported neurological symptom. At standard examination, 42/105 (40\%) exhibited neurological abnormalities, including subjective dysgeusia/ hyposmia $(n=19)$, enhanced physiological tremor $(n=15)$ and cognitive impairment $(n=17)$. Two patients exhibited isolated feet hypoesthesia and two reached a diagnosis of sensor-motor 
Table 1 Demographic and clinical characteristics of the sample according to COVID-19 severity

\begin{tabular}{|c|c|c|c|c|c|}
\hline & Total $(n=165)$ & Mild ( $n=57)$ & Moderate $(n=77)$ & Severe $(n=31)$ & $p$ value \\
\hline Age, years & $64.8 \pm 12.6$ & $62.3 \pm 13.1$ & $67.3 \pm 12.3$ & $63.2 \pm 11.2$ & 0.06 \\
\hline Sex, female $n(\%)$ & $50(30.3 \%)$ & $22(38.5 \%)$ & $18(23.4 \%)$ & $10(32.2 \%)$ & 0.16 \\
\hline Days of hospitalisation & $11.6 \pm 8.8$ & $7.8 \pm 4.2$ & $12.3 \pm 9.4$ & $17.1 \pm 10.4$ & $0.001 \# q *$ \\
\hline Oxygen therapy, $n(\%)$ & $128(77.6 \%)$ & $20(35.1 \%)$ & $77(100 \%)$ & $31(100 \%)$ & 0.0019 \\
\hline $\begin{array}{l}\text { Non-invasive ventilation, } \\
n(\%)\end{array}$ & $18(11.0 \%)$ & $0(0 \%)$ & $0(0 \%)$ & $18(58.3 \%)$ & $0.001 \# \Phi$ \\
\hline Intubation, $n(\%)$ & $2(1.2 \%)$ & $0(0 \%)$ & $0(0 \%)$ & $2(6.4 \%)$ & $0.001 \# q$ \\
\hline qSOFA & $0.44 \pm 0.53$ & $0.0 \pm 0.0$ & $0.48 \pm 0.50$ & $1.10 \pm 0.29$ & $0.001 \# q *$ \\
\hline CIRS pre-total & $18.6 \pm 3.3$ & $18.1 \pm 3.2$ & $19.4 \pm 3.6$ & $17.9 \pm 2.8$ & 0.03 \\
\hline CIRS, pre-severity mean & $1.35 \pm 0.25$ & $1.31 \pm 0.24$ & $1.40 \pm 0.26$ & $1.28 \pm 0.20$ & 0.02 \\
\hline
\end{tabular}

*Significant for the comparison between mild vs moderate groups

\#Significant for the comparison between moderate vs severe groups

qSignificant for the comparison between mild vs severe groups polyneuropathy, with distal mild sensory and motor deficits (bilateral deficits within the extension of feet 4/5 MRC) confirmed by electrophysiological study. No alterations within cranial nerves, cerebellar function or pyramidal signs were detected.

Neurological abnormalities at the examination were associated with older age $(p=0.005)$, higher number of premorbid comorbidities $(p=0.001)$, higher COVID-19 severity $(p=0.05)$, longer hospitalisation $(p=0.002)$ and higher number of neurological symptoms reported $(p=0.007)$. Logistic regression analyses adjusted for age and sex identified duration of hospitalisation $(p=0.02, \operatorname{Exp}(B) 1.19)$ and premorbid comorbidities $(p=0.03, \operatorname{Exp}(B) 1.19)$ as the best predictors of neurological abnormalities at examination.

\section{Discussion}

Findings showed that previously hospitalised COVID-19 patients reported a wide array of neurological symptoms 6 months after SARS-CoV-2 infection, predicted by combination of age, premorbid conditions and severity of the disease.

\begin{tabular}{|c|c|c|c|c|}
\hline \multirow{2}{*}{\multicolumn{2}{|c|}{$\begin{array}{l}\text { Symptoms reported at 6-months } \\
\qquad \text { Total }(n=165)\end{array}$}} & \multicolumn{3}{|c|}{ Symptoms according to COVID-19 severity } \\
\hline & & Mild $(n=57)$ & Moderate $(n=77)$ & Severe $(n=31)$ \\
\hline Fatigue & $33,9 \%$ & $29,8 \%$ & $31,2 \%$ & $48,4 \%$ \\
\hline Memory/Concentration complaints & $31,5 \%$ & \begin{tabular}{|l|}
$33,3 \%$ \\
\end{tabular} & $20,8 \%$ & $48,4 \%$ \\
\hline Sleep disorders & $31,5 \%$ & \begin{tabular}{|l} 
\\
$43,8 \%$
\end{tabular} & $19,5 \%$ & $38,7 \%$ \\
\hline Myalgia & $30,3 \%$ & $31,6 \%$ & $24,7 \%$ & $38,7 \%$ \\
\hline Depressive symptoms/ anxiety & $26,7 \%$ & $33,3 \%$ & $19,5 \%$ & $32,3 \%$ \\
\hline Dyspnea & $21,2 \%$ & $15,0 \%$ & $22,1 \%$ & $29,0 \%$ \\
\hline Loss of dependency in IADL & $20,7 \%$ & $17,5 \%$ & $38,2 \%$ & $32,4 \%$ \\
\hline Blurring/loss of vision & $19,5 \%$ & \begin{tabular}{|l|l}
$19,3 \%$ \\
\end{tabular} & $11,7 \%$ & $38,7 \%$ \\
\hline Numbness/tingling & $18,8 \%$ & \begin{tabular}{|l|l|}
$21,4 \%$ \\
\end{tabular} & $15,6 \%$ & $22,6 \%$ \\
\hline Hyposmia/hypogeusia & $16,4 \%$ & $12,3 \%$ & $15,6 \%$ & $25,8 \%$ \\
\hline Urinary dysfunction & $13,9 \%$ & $15,8 \%$ & $11,7 \%$ & $16,1 \%$ \\
\hline Confusion & $13,3 \%$ & $12,3 \%$ & $9,1 \%$ & $25,8 \%$ \\
\hline Hypotension & $12,2 \%$ & $17,5 \%$ & $5,2 \%$ & $19,4 \%$ \\
\hline Gait disturbances & $10,9 \%$ & $14,0 \%$ & $7,8 \%$ & $12,9 \%$ \\
\hline Abnormal movements & $10,3 \%$ & $12,3 \%$ & $6,5 \%$ & $16,1 \%$ \\
\hline Headache & $9,7 \%$ & $12,5 \%$ & $6,5 \%$ & $12,9 \%$ \\
\hline Postural instability or falls & $8,5 \%$ & $7,0 \%$ & $6,5 \%$ & $16,1 \%$ \\
\hline Swalowing difficulties & $6,1 \%$ & $7,0 \%$ & $2,6 \%$ & $12,9 \%$ \\
\hline
\end{tabular}

Fig. 1 Prevalence of symptoms at 6 months follow-up in the whole samples and in subgroups of patients stratified by COVID-19 severity. Abnormal movements included tremor, dystonia, dyskinesia, chorea and all hyperkinetic disorders 
These data extend recent studies which have argued for a high prevalence of post-COVID clinical manifestations and claimed that long-term consequences of COVID-19 involve both central and peripheral nervous systems [6-8, 13, 14].

In the present cohort of patients with 6 months of follow-up and extensive neurological evaluation, the most prevalent symptoms reported were fatigue, memory complaints, sleep disorders and myalgias followed by depression/anxiety, visual disturbances, paraesthesia and hyposmia. These findings extended the earlier works of Carfi [7] and Goertz [8], reporting a high persistence of respiratory symptoms and fatigue in more than half of hospitalised and non-hospitalised patients 3 months after COVID-19. In our study, long-term neurological complaints showed different distributions according to COVID-19 severity, which was associated with an increased total number of symptoms, fatigue, memory complaints and confusion. The impact of SARS-CoV-2 severity in long-term persistent respiratory symptoms and fatigue has been recently highlighted by a large survey 6 months after discharge in Wuhan [6]. Our study, conducted in smaller but more homogeneous older population consecutively enrolled in a single COVID-19 unit, suggests that even small differences in the severity of infection can impact long-term neurological manifestations. This effect was prominent for cognitive symptoms, including memory complaints and attention deficitswhereas other symptoms - such as myalgias or numbness appeared to be independent of severity. This might suggest a higher vulnerability of the central nervous system to severe SARS-CoV-2 infection possibly through higher general inflammatory response and longer hospitalisation - as already highlighted for other infectious diseases [15]. We found age and premorbid comorbidities as important independent predictors of long-term symptoms, at variance with the data reported by Huang and coauthors for respiratory symptoms and fatigue [6]. These findings are of particular interest, as we excluded patients with premorbid dementia or other known neurological conditions or patients with neurologic COVID19 presentation [5], focusing on neurological features in patients with prominent respiratory COVID-19 disease [16].

At standardised neurological examination, $40 \%$ of subjects exhibited mild abnormalities-not reported before the hospitalisation for COVID-19 disease. The most prevalent features were hyposmia, cognitive impairment, enhanced physiological tremor and subtle motor and sensory low-limb deficits in several cases. Age, premorbid comorbidities and longer hospitalisation were, again, the strongest predictors of neurological abnormalities at examination, notwithstanding if the numbers did not allow separate analyses for different neurological features. This would suggest that SARS-CoV-2 infection has probably a stronger long-term neurological impact in older subjects with higher vulnerability who suffered an acute respiratory syndrome. The complex interaction between physical and brain resilience and long-term disability after hospitalisation has been indeed already demonstrated for other infectious diseases, such as community-acquired pneumonia [15]. In fact, we need to acknowledge that the experience of hospitalisation due to COVID-19 in frail elderly should is an important confounding factor-as it can influence the relationship between long-term manifestations and psychosocial long-term disturbances [17-19].

Several limitations should be acknowledged. First, premorbid conditions were based on medical records and assessment during hospitalisation thus not allowing a complete premorbid neurological screening. Second, we excluded from the study patients with neurological disorders before or concomitant the acute phase of SARS-CoV-2 infection, thus potentially underestimating the global neurological burden due to COVID-19. Furthermore, this is a single-centre study conducted in an homogeneous cohort of patients with moderate severity of COVID-19 and large studies including intubated and non-hospitalised patients are warranted to confirm these findings.

Limitations notwithstanding, our findings indicate that several neurological features are a relevant component of long-term manifestations of COVID-19 disease especially in more vulnerable and severe patients, thus underlying the clinical need for longitudinal programmes able to track the real impact of SARS-CoV-2 infection on long-term brain health status [18-21].

Supplementary Information The online version contains supplementary material available at https://doi.org/10.1007/s10072-021-05586-4.

Acknowledgements The authors thank all the participants for their participation.

Author contribution Conception and design of the study: AP, VC, ML, DS and AP. Acquisition and analysis of data: AP, VC, SCP, NZ, GB, AC, SG, IL, DC, MF, MB and AP.

Drafting the manuscript and figures: AP, VC, GB and AP.

Funding Open access funding provided by Università degli Studi di Brescia within the CRUI-CARE Agreement.

Data availability The data that support the findings of this study are available from the corresponding author upon reasonable request.

\section{Declarations}

Conflict of interest Andrea Pilotto served on the advisory board of Z-cube (technology division of Zambon pharmaceuticals); he received honoraria from Z-cube s.r.l., Biomarin, Zambon, Abbvie, Nutricia and Chiesi pharmaceuticals. He received research support from Vitaflo Germany and Zambon Italy.

Viviana Cristillo has no financial conflicts to disclose.

Stefano Cotti Piccinelli has no financial conflicts to disclose.

Nicola Zoppi has no financial conflicts to disclose.

Giulio Bonzi has no financial conflicts to disclose.

Davide Sattin has no financial conflicts to disclose.

Silvia Schiavolin has no financial conflicts to disclose.

Alberto Raggi has no financial conflicts to disclose.

Antonio Canale has no financial conflicts to disclose.

Stefano Gipponi has no financial conflicts to disclose. 
Ilenia Libri has no financial conflicts to disclose.

Martina Frigerio has no financial conflicts to disclose.

Michela Bezzi has no financial conflicts to disclose.

Matilde Leonardi has no financial conflicts to disclose.

Alessandro Padovani is consultant and served on the scientific advisory board of GE Healthcare, Eli-Lilly and Actelion Ltd. Pharmaceuticals and received speaker honoraria from Nutricia, PIAM, Langstone Technology, GE Healthcare, Lilly, UCB Pharma and Chiesi Pharmaceuticals. He is funded by grant of the Ministry of University (MURST).

Ethical approval The study was approved by the local ethics committee of ASST "Spedali Civili di Brescia" Hospital and the requirement for informed consent was waived by the Ethics Commission (NP 4166).

Open Access This article is licensed under a Creative Commons Attribution 4.0 International License, which permits use, sharing, adaptation, distribution and reproduction in any medium or format, as long as you give appropriate credit to the original author(s) and the source, provide a link to the Creative Commons licence, and indicate if changes were made. The images or other third party material in this article are included in the article's Creative Commons licence, unless indicated otherwise in a credit line to the material. If material is not included in the article's Creative Commons licence and your intended use is not permitted by statutory regulation or exceeds the permitted use, you will need to obtain permission directly from the copyright holder. To view a copy of this licence, visit http://creativecommons.org/licenses/by/4.0/.

\section{References}

1. Saglietto A, D'Ascenzo F, Zoccai GB, de Ferrari GM (2020) COVID-19 in Europe: the Italian lesson. Lancet 395(10230): 1110-1111. https://doi.org/10.1016/S0140-6736(20)30690-5

2. Paterson RW, Brown RL, Benjamin L, Nortley R, Wiethoff S, Bharucha T, Jayaseelan DL, Kumar G, Raftopoulos RE, Zambreanu L, Vivekanandam V, Khoo A, Geraldes R, Chinthapalli K, Boyd E, Tuzlali H, Price G, Christofi G, Morrow $\mathrm{J}$ et al (2020) The emerging spectrum of COVID 19 neurology: clinical, radiological and laboratory findings. Brain 143(10): 3104-3120. https://doi.org/10.1093/brain/awaa240

3. Haidar Jourdi H, Hai Hassan Z et al (2021) Neurological and neuropsychological changes associated with SARS-CoV-2 infection: new observations, new mechanisms. Neuroscientist 1073858420984106. doi: https://doi.org/10.1177/1073858420984106.

4. Romoli M, Jelcic I, Bernard-Valnet R, García Azorín D, Mancinelli L, Akhvlediani T, Monaco S, Taba P, Sellner J, for the Infectious Disease Panel of the European Academy of Neurology (2020) Infectious disease panel of the European Academy of Neurology. A systematic review of neurological manifestations of SARS-CoV-2 infection: the devil is hidden in the details. Eur J Neurol. 27(9):1712-1726

5. Pilotto A, Benussi A, Libri I, Masciocchi S, Poli L, Premi E, Alberici A, Baldelli E, Bonacina S, Brambilla L, Benini M, Caratozzolo S, Cortinovis M, Costa A, Cotti Piccinelli S, Cottini E, Cristillo V, Delrio I, Filosto M et al (2021) COVID-19 impact on consecutive neurological patients admitted to the emergency department. J Neurol Neurosurg Psychiatry 92(2):218-220. https://doi.org/10.1136/jnnp2020-323929

6. Huang C, Huang L, Wang Y, Li X, Ren L, Gu X, Kang L, Guo L, Liu M, Zhou X, Luo J, Huang Z, Tu S, Zhao Y, Chen L, Xu D, Li Y, Li C, Peng L et al (2021) 6-month consequences of COVID-19 in patients discharged from hospital: a cohort study. Lancet 397(10270):220-232. https://doi.org/10.1016/S0140-6736(20)32656-8

7. Carfi A, Bernabei R, Landi F (2020) Persistent symptoms in patients after acute. COVID-19 JAMA 324(6):603-605. https://doi. org/10.1001/jama.2020.12603

8. Goërtz YMJ, Van Herck M, Delbressine JM et al (2020) Persistent symptoms 3 months after a SARS-CoV-2 infection: the postCOVID-19 syndrome? ERJ Open Res 6:00542-02020

9. Linn BS, Linn MW, Gurel L (1968) Cumulative illness rating scale. J Am Geriatr Soc 16(5):622-626. https://doi.org/10.1111/j.15325415.1968.tb02103.x

10. Piva S, Filippini M, Turla F, Cattaneo S, Margola A, de Fulviis S, Nardiello I, Beretta A, Ferrari L, Trotta R, Erbici G, Focà E, Castelli F, Rasulo F, Lanspa MJ, Latronico N (2020) Clinical presentation and initial management critically ill patients with severe acute respiratory syndrome coronavirus 2 (SARS-CoV-2) infection in Brescia, Italy. J Crit Care 58:29-33

11. Zhou F, Yu T, Du R et al (2020) Clinical course and risk factors for mortality of adult inpatients with COVID-19 in Wuhan, China: a retrospective cohort study. Lancet 395:1054-1062

12. Santangelo G, Siciliano M, Pedone R, Vitale C, Falco F, Bisogno R, Siano P, Barone P, Grossi D, Santangelo F, Trojano L (2015) Normative data for the. Montreal Cognitive Assessment in an Italian population sample Neurol Scie 36(4):585-591. https://doi. org/10.1007/s10072-014-1995-y

13. Jarrahi A, Ahluwalia M, Khodadadi H et al (2020) Neurological consequences of COVID-19: what have we learned and where do we go from here? J Neuroinflamm 17(1):286

14. Matschke J, Lütgehetmann M, Hagel C et al (2020) Neuropathology of patients with COVID-19 in Germany: a postmortem case series. Lancet Neurol 0:1-11

15. Metlay JP, Fine MJ, Schulz R, Marrie TJ, Coley CM, Kapoor WN, Singer DE (1997) Measuring symptomatic and functional recovery in patients with community-acquired pneumonia. J Gen Intern Med 12(7):423-430. https://doi.org/10.1046/j.1525-1497.1997.00074.x

16. Townsend L, Dyer AH, Jones K, Dunne J, Mooney A, Gaffney F, O'Connor L, Leavy D, O'Brien K, Dowds J, Sugrue JA, Hopkins D, Martin-Loeches I, Ni Cheallaigh C, Nadarajan P, McLaughlin AM, Bourke NM, Bergin C, O'Farrelly C et al (2020) Persistent fatigue following SARS-CoV-2 infection is common and independent of severity of initial infection. PLOS ONE 15(11):e0240784. https:// doi.org/10.1371/journal.pone.0240784

17. Holmes EA, O’Connor RC, Perry VH et al (2020) Multidisciplinary research priorities for the COVID-19 pandemic: a call for action for mental health science. Lancet Psychiatry 7(6):547-560

18. Taquet M, Luciano S, Geddes J, Harrison PJ (2020) Bidirectional associations between COVID-19 and psychiatric disorder: retrospective cohort studies of 62354 COVID-19 cases in the USA. Lancet Psychiatry S2215-0366(20):30462-30464. https://oi.org/ $10.1016 / \mathrm{S} 2215-0366(20) 30462-4$

19. Miners S, Kehoe PG, Love S (2020) Cognitive impact of COVID19: looking beyond the short term. Alzheimers Res Ther. 2020;12 (1):170. doi: https://doi.org/10.1186/s13195-020-00744-w.

20. Stam HJ, Stucki G, Bickenbach J European Academy of Rehabilitation Medicine (2020) Covid-19 and post intensive care syndrome: a call for action. J Rehabil Med 52(4):jrm00044. https:// doi.org/10.2340/16501977-2677

21. Greenhalgh T, Knight M, A'Court C et al (2020) Management of post-acute covid-19 in primary care. Management of post-acute covid-19 in primary care. BMJ. 2020 370:m3026.

Publisher's note Springer Nature remains neutral with regard to jurisdictional claims in published maps and institutional affiliations. 